\title{
Application of Fractional Scaling in Modelling of Magnetic Power Losses
}

\author{
M. NAJGEBAUER* \\ Częstochowa University of Technology, Faculty of Electrical Engineering, \\ al. Armii Krajowej 17, 42-200 Częstochowa, Poland \\ (Received February 2, 2015)
}

\begin{abstract}
The paper presents a new approach to the Widom-based scaling procedure, in which additional fractional exponents were introduced into the Maclaurin series. The modified scaling procedure was proposed in order to obtain more universal descriptions in a form of the power law series with fractional exponents. The proposed procedure was examined for the power losses scaling of commercial grain-oriented electrical steel.
\end{abstract}

DOI: $10.12693 /$ APhysPolA.128.107

PACS: 75.50.Bb, 75.60.Ej, 89.75.Da

\section{Introduction}

The scaling hypothesis has been considered independently by Widom, Kadanoff and other scientists for analysis of critical phenomena and phase transitions. It provided two prediction categories as scaling laws and data collapse [1]. The scaling laws are universal relationships bounding scaling exponents, whereas the data collapse is a transformation, which allows one to reduce a plane of data sets to a single curve, represented in scaled coordinates. The investigation of scaling behaviour for various phenomena, also far from the critical point, is still the field of theoretical and applied research, including material sciences.

In this paper, the modified Widom-based scaling procedure with fractional exponents of the Maclaurin series is proposed. The proposed approach should allow one to obtain more universal formulae as well as to reduce the number of power law terms necessary to obtain the data collapse. These assumptions are examined for the power losses scaling of commercial grain-oriented electrical steel.

\section{The Widom-like scaling procedure}

The scaling procedure proposed by Widom [2] assumes that the equation of state, describing thermodynamical phenomenon and given by a functional relationship $Z=f(X, Y)$, is the generalised homogeneous function defined as:

$$
\exists a, b, c: \forall \lambda>0, \quad \lambda^{a} Z=f\left(\lambda^{b} X, \lambda^{c} Y\right) .
$$

For a appropriate choice of the coefficient $\lambda=Y^{-1 / c}$, the relationship (1) can be transformed as follows:

$$
Y^{-\alpha} Z=f\left(Y^{-\beta} X, 1\right),
$$

and finally reduced to the scaled form

\footnotetext{
* e-mail: najgebauer@el.pcz.czest.pl
}

$$
Z_{Y}=F\left(X_{Y}\right),
$$

where: $Z_{Y}=Z / Y^{\alpha}, X_{Y}=X / Y^{\beta}$ are the scaled variables, $\alpha=a / c, \beta=b / c$ are the scaling exponents and $F(x)=f(x, 1)$ is called a scaling function [1]. After extension the right side of (2) into Maclaurin series, the following relationship is obtained:

$$
Z_{Y}\left(X_{Y}\right)=F_{0}+F_{1} X_{Y}+F_{2} X_{Y}^{2}+F_{3} X_{Y}^{3}+\ldots,
$$

where $F_{n}$ are the scaling factors. It should be stated that the variable $Z_{Y}$ is given by the series of power laws with integer exponents of independent variable $X_{Y}$. It is some kind of limitation, because many phenomena are described by power laws with fractional exponents. In order to improve the scaling procedure, the substitution $X_{Y} \rightarrow X_{Y}^{x}$ is proposed, which results in the modified relationship

$$
Z_{Y}\left(X_{Y}^{x}\right)=F_{0}+F_{1} X_{Y}^{x}+F_{2} X_{Y}^{2 x}+F_{3} X_{Y}^{3 x}+\ldots
$$

where $x$ is the fractional exponent. It should be noted that for $x=1$ the relationship (4) is reduced to the (3) one. Thereby, the relationship (4) has more universal nature.

Previously Ruszczyk et al. [3, 4] have proposed the Maclaurin series with non-integer exponents given by $i(1-x)$, where $i=1,2,3 \ldots$ are integer exponents of the series and $x$ is the tuning parameter. This approach was applied in the scaling of core losses in soft magnetic materials exposed to non-sinusoidal flux wave forms and DC bias conditions in order to improve the multi-parameters model fitting to scaled measurement data by introducing a natural correction of the Maclaurin series' exponents via the tuning parameter.

In the present paper the fractional exponent $x$ is a free parameter, which is considered in the Maclaurin series not for improvement of the fitting procedure, but in order to provide a more universal and flexible formula, describing scaling behaviours of phenomena. Moreover, it is also assumed that the use of the fractional scaling procedure should result in reduction of the number of power law terms, which are necessary to obtain the data collapse. The abovementioned assumption was made, 
because there are plenty of models in the form of a single power law with a fractional exponent, used in different fields of science.

The proposed Widom-based scaling procedure with fractional exponents of the Maclaurin series is examined in this paper for the power loss scaling of commercial grain-oriented electrical steel.

\section{Power laws in losses modelling for soft magnetic materials}

Power losses in soft magnetic materials are related to magnetic hysteresis phenomenon and eddy currents flows. Their modelling is a field of research for many scientists and engineers. The most popular model of power losses has been proposed by Bertotti [5-7]. This model is composed of three power laws of magnetic induction $B_{\mathrm{m}}$ and frequency $f$ :

$$
P_{\text {tot }}=c_{1} B_{\mathrm{m}}^{k} f+c_{2}\left(B_{\mathrm{m}} f\right)^{2}+c_{3}\left(B_{\mathrm{m}} f\right)^{3 / 2},
$$

and the following power laws represent hysteresis loss, eddy currents loss and excess loss, respectively. This approach is known as the power losses separation. The Bertotti formula does not always give satisfactory results, especially in modelling of excess loss for magnetic materials with amorphous, micro- and nanocrystalline structure $[8,9]$.

It should be noted that in 1972 Culity suggested that the losses separation in soft magnetic materials is an artificial process and thereby power losses should be considered as total. It indicates that power losses in soft magnetic materials might be described by a simply, single power law of magnetic induction and frequency. The simple models of power losses in the form of a single power law with non-integer exponents $\alpha$ and $\beta$ :

$$
P_{\text {tot }}=k B_{\mathrm{m}}^{\beta} f^{\alpha} \text {, }
$$

was proposed in [10-16], including the author's previous paper [14]. Also some technical reports provide similar empirical formulae for power losses $[17,18]$. The aforementioned considerations were a direct cause for staring the study on application the scaling procedure with fractional exponents of the Maclaurin series in investigation of scaling behaviour of power losses in soft magnetic materials.

\section{Fractional scaling of power losses - results and discussion}

The scaling procedure with fractional exponents of the Maclaurin series (the fractional scaling procedure) has been examined for the power loss scaling of commercial grain-oriented electrical steel, grade 111-35-N5. The sample was a single square sheet $500 \times 500 \times 0.35 \mathrm{~mm}$ in dimensions. Power losses were measured at frequency range form $10 \mathrm{~Hz}$ up to $500 \mathrm{~Hz}$ and for induction with amplitude varying from 0.1 to $1.8 \mathrm{~T}$ with $0.1 \mathrm{~T}$ step. The measurements ware carried out at Electrotechnical Institute, Wrocław using the computer-aided Single Sheet Tester MAG-RJJ-2.0, according to the international IEC 404-3 Standard (sinusoidal waveform of magnetic induction inside the sample is required) [19].
Previously, the scaling procedure with integer exponents of the Maclaurin series has been examined for various types of soft magnetic materials [20-26], which provided the two-term power law

$$
P_{B}=p_{1} f_{B}+p_{2} f_{B}^{2} .
$$

In the case of the fractional scaling procedure given by (4), a three-term formula for the power loss scaling was proposed

$$
P_{B}=p_{1} f_{B}^{x}+p_{2} f_{B}^{2 x}+p_{3} f_{B}^{3 x},
$$

where $p_{1}, p_{2}$ are the scaling factors, $x$ is the fractional exponent and $P_{B}, f_{B}$ denote power losses and frequency, scaled with respect to induction $B$, respectively

$$
P_{B}=\frac{P_{\mathrm{tot}}}{B_{\mathrm{m}}^{\beta}}, \quad f_{B}=\frac{f}{B_{\mathrm{m}}^{\alpha}} .
$$

The parameter values $\left(p_{1}, p_{2}, p_{3}, \alpha, \beta\right.$ and $\left.x\right)$ of the formula (8) were estimated from measurement data using the least-squares method, implemented in the Microsoft Excel Solver tool with the Generalized Reduced Gradient method of nonlinear optimization. In order to evaluate influence of the number of power law terms on results of the losses scaling, the parameters estimation was also made for the reduced formulae (8), composed of threeterms, two-terms and one-term power laws, respectively

$$
\begin{aligned}
& P_{B}=p_{1} f_{B}^{x}+p_{2} f_{B}^{2 x}+p_{3} f_{B}^{3 x}, \\
& P_{B}=p_{1} f_{B}^{x}+p_{2} f_{B}^{2 x}, \\
& P_{B}=p_{1} f_{B}^{x} .
\end{aligned}
$$

The results of the power losses scaling (i.e. the data collapse of losses) of grain-oriented steel for the fractional scaling procedures are depicted in Figs. 1-3 and compared to results of the integer scaling procedure, depicted in Fig. 4. Values of the estimated parameters as well as mean percentage errors (MPE) between theoretical and experimental values of the scaled losses obtained for the considered formulae (10)-(12) are presented in Table.

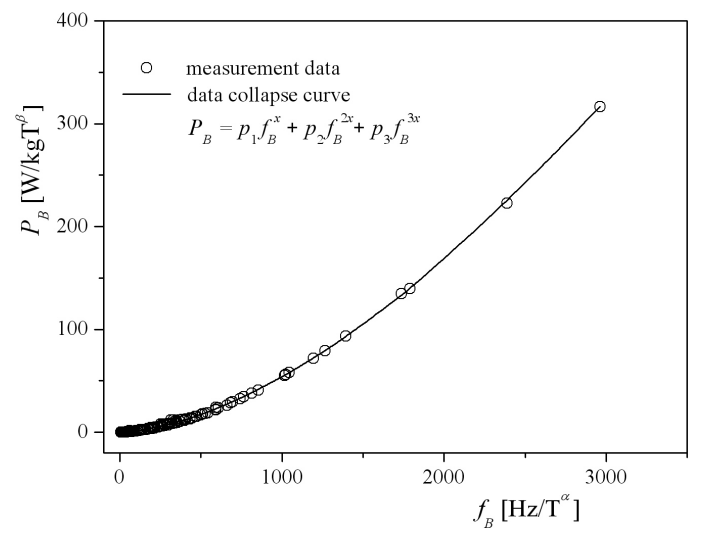

Fig. 1. Data collapse of power losses for the scaling procedure with fractional exponents (3-terms formula).

The proposed fractional scaling procedures make it possible to investigate the scaling behaviour of power losses in grain-oriented steel. The satisfactory results of the power losses scaling are obtained for all considered formulae (10)-(12). It should be noted that graphical 


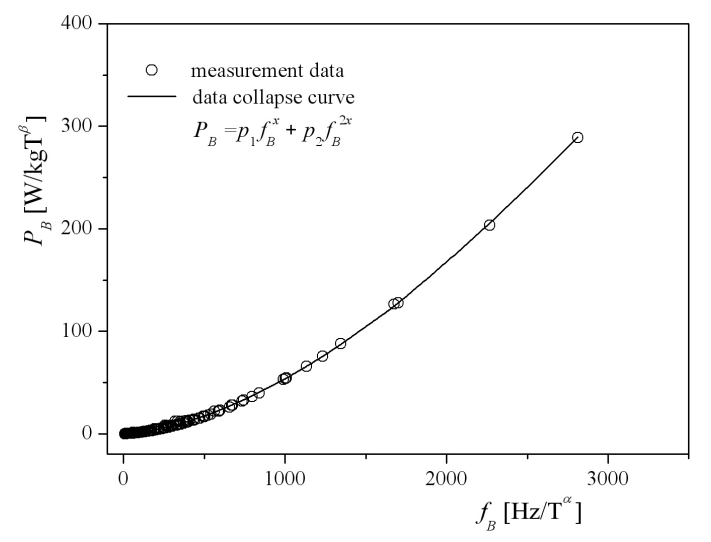

Fig. 2. As in Fig. 1 but for 2-terms formula.

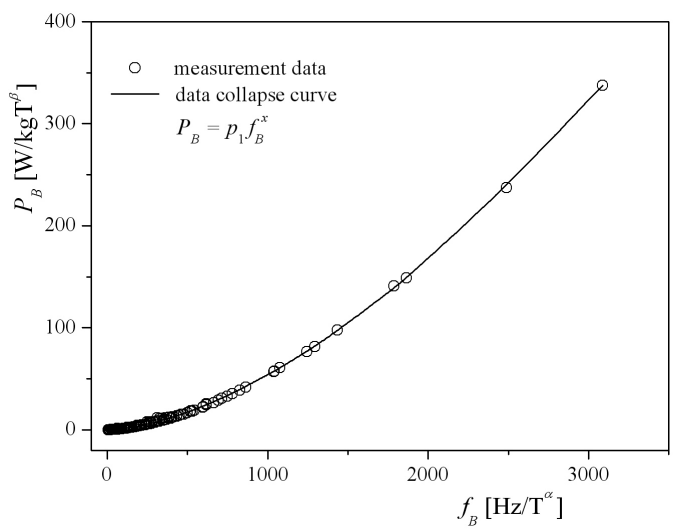

Fig. 3. As in Fig. 1 but for 1-term formula.

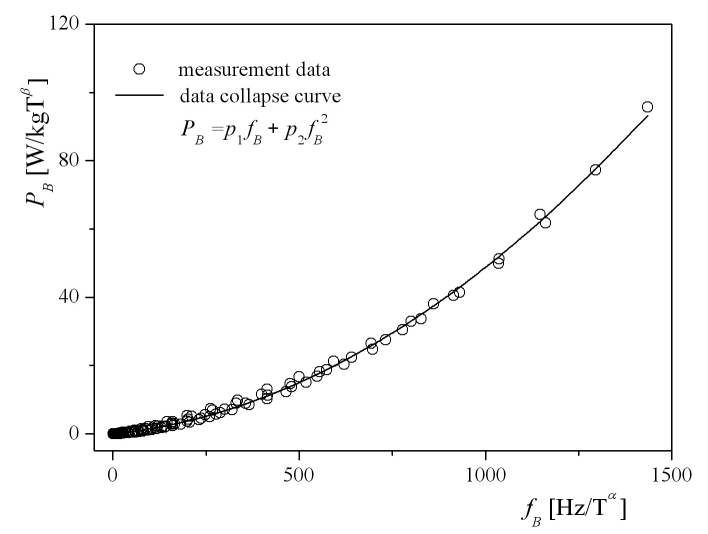

Fig. 4. Data collapse of power losses for the scaling procedure with integer exponents.

TABLE

Values of the estimated parameters and the mean square errors (MPE) of the fractional scaling of power losses for grain-oriented electrical steel, grade 111-35-N5.

\begin{tabular}{c|c|c|c|c|c|c|c}
\hline \hline $\begin{array}{c}\text { Formula } \\
\text { structure }\end{array}$ & $\alpha$ & $\beta$ & $x$ & $\begin{array}{c}p_{1} \\
{\left[\frac{\mathrm{W}}{\mathrm{kg}} \frac{T^{\alpha-\beta}}{\mathrm{Hz}}\right]}\end{array}$ & $\left.\begin{array}{c}p_{2} \\
{\left[\frac{\mathrm{W}}{\mathrm{kg}} \frac{T^{2 \alpha-\beta}}{\mathrm{Hz}}\right]}\end{array}\right]$ & $\left.\begin{array}{c}p_{3} \\
{\left[\frac{\mathrm{W}}{\mathrm{kg}} \frac{T^{3 \alpha-\beta}}{\mathrm{Hz}}\right.}\end{array}\right]$ & $\begin{array}{c}\mathrm{MPE} \\
{[\%]}\end{array}$ \\
\hline 3 terms & 3.19 & 0.81 & 0.52 & $9.48 \times 10^{-2}$ & $-1.03 \times 10^{-2}$ & $1.26 \times 10^{-3}$ & 10.16 \\
2 terms & 3.21 & 0.83 & 0.81 & $1.83 \times 10^{-3}$ & $-6.73 \times 10^{-4}$ & - & 7.12 \\
1 term & 3.17 & 0.79 & 1.63 & $7.00 \times 10^{-4}$ & - & - & 8.44
\end{tabular}

representations of the scaling results and values of the mean percentage errors are similar in all analysed cases. Thus, it might be stated that the fractional scaling procedure in the form of a single power law is the best solution, because it provides the comparable scaling results for the lowest number of parameters. The use of successive power laws, related to higher terms of the Maclaurin series, did not improve results of the losses scaling significantly. Therefore, their application in the considered scaling procedure is redundant.

The scaling exponents $\alpha$ and $\beta$ have comparable values in all analysed cases, which might suggest that these are specific parameters, characteristic for the tested material. Values of the other parameters are different, depending on formulae used in the losses scaling. Thus, these parameters are rather related to the estimation procedure than to the materials properties. However in the case of the single power law procedure, a value of the fractional exponent $x$ is between the values characteristic for the frequency dependences of hysteresis losses $(x=1)$ and eddy-current losses $(x=2)$. It might suggest that the fractional exponent should be also considered as a material parameter, because its value might indicate a contribution of different dissipation mechanisms in total power losses, as well. However, this assumption requires further research concerning the power loss scaling for soft magnetic materials with internal structures different from the grain-oriented crystalline one.

5. Fractional scaling in modelling of power losses

The proposed relationship of the scaled power losses, given in the form of a single power law (12), can be easily transformed to the formula, directly describing the total power losses

$$
P_{B}=p f_{B}^{x}, \quad \frac{P_{\mathrm{tot}}}{B_{\mathrm{m}}^{\beta}}=p\left(\frac{f}{B_{\mathrm{m}}^{\alpha}}\right)^{x}, \quad P_{\mathrm{tot}}=p \frac{B_{\mathrm{m}}^{\beta}}{B_{\mathrm{m}}^{\alpha x}} f^{x},
$$

and finally

$$
P_{\mathrm{tot}}=p B_{\mathrm{m}}^{y} f^{x},
$$

where $y=\beta-\alpha x$. The relationship (13) has the form, which is equivalent to the currently used models of power losses in the form of a single power law with non-integer exponents, given by Eq. (6).

The relationship (13) was used in power losses modelling of commercial grain-oriented steel 111-35-N2 and for previously estimated parameters $(p, \alpha, \beta$ and $x)$, given in Table. The modelling results of the power losses frequency dependences for chosen values of magnetic induction are depicted in Fig. 5. The average errors of the power losses modelling are equal to $4.26 \%, 1.52 \%$, and $7.62 \%$ for $B_{\mathrm{m}}=0.5,1.0$, and $1.5 \mathrm{~T}$, respectively. The maximum errors of the power losses modelling do not exceed $12 \%$.

The results obtained for grain-oriented steel prove that the fractional scaling procedure is a useful tool in the power losses modelling, which provides modelling errors on acceptable level. The proposed approach to the power losses modelling will be examined for other types of commercial soft magnetic materials. 


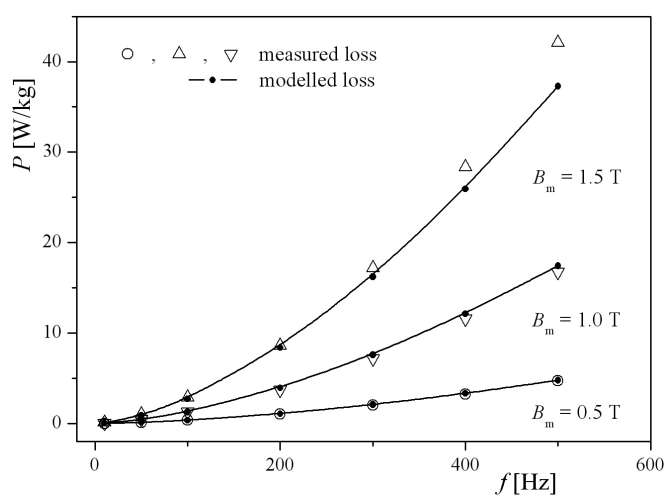

Fig. 5. Power losses versus frequency for commercial grain-oriented steel 111-35-N2.

\section{Conclusions}

The modified Widom-like scaling procedure with fractional exponents of the Maclaurin series has been proposed. It was assumed that the use of this procedure should make scaling formulae more universal as well as it should reduce the number of power law terms necessary for collapse of measurement data. These assumptions were verified for power losses in grain-oriented steel. The proposed scaling procedure resulted in the simple formula for power losses in the form of a single power law, which provided scaling results comparable with more expanded formulae. The obtained results confirm the initial assumptions as well as reveal the scaling behaviour of power losses in grain-oriented steel. The proposed scaling formula was also applied in the modelling of power losses. The scaling-based modelling of power losses gave promising results and it will be the field of further considerations.

The proposed scaling procedure with fractional exponents of the Maclaurin series will be examined in the power losses scaling for various types of soft magnetic materials. Further research will be also conducted to determine relationships between the scaling exponents $\alpha$ and $\beta$ as well as to investigate the role of the fractional exponent $x$ as an indicator of energy dissipation mechanisms in soft magnetic materials. Moreover, the fractional scaling procedure will be used in investigations of the scaling behaviour of other magnetic properties.

\section{Acknowledgments}

Author would like to thank Prof. Jan Szczygłowski for providing measurement data of power losses for grainoriented steel and Prof. Krzysztof Sokalski for interesting discussions.

\section{References}

[1] H.E. Stanley, Rev. Mod. Phys. 71, S358 (1999).

[2] B. Widom, J. Chem. Phys. 43, 3898 (1965).

[3] A. Ruszczyk, K. Sokalski, J. Szczygłowski, arXiv:1309.0022.

[4] A. Ruszczyk, K. Sokalski, COMPEL 34, 371 (2015).
[5] G. Bertotti, Hysteresis in Magnetism, Academic Press, San Diego 1998.

[6] G. Bertotti, J. Magn. Magn. Mater. 41, 253 (1984).

[7] G. Bertotti, IEEE Trans. Magn. 24, 621 (1998).

[8] G.E. Fish, C.-F. Chang, J. Appl. Phys. 64, 5370 (1988).

[9] J. Szczygłowski, P. Kopciuszewski, W. Wilczyński, A. Roman, Mater. Sci. Eng. B 75, 13 (2000).

[10] R.M. Bozorth, Ferromagnetism, IEEE Press, New York 1993.

[11] B.D. Culity, C.D. Graham, Introduction to Magnetic Materials, Addison-Wesley, 1972 (2nd ed. WileyIEEE Press, New Jersey 2009).

[12] E.C. Snelling, Soft Ferrities. Properties and Applications, 2nd ed., Butterworths, London 1988.

[13] S. Mulder, Power Conv. Intellig. Motion 21, 22 (1995).

[14] M. Najgebauer, K. Chwastek, J. Szczygłowski, P. Kopciuszewski, in: Proc. Soft Magnetic Materials, Düsseldorf (Germany), 2004, Vol. 16, Ed. D. Raabe, Max-Planck Institut für Eisenforschung GmbH, Düssseldorf 2004, p. 221.

[15] K. Sokalski, J. Szczygłowski, W. Wilczyński, Int. J. Condens. Matter 12, 1 (2014).

[16] J. Szczygłowski, Novel Soft Magnetic Materials. Chosen Properties, Wydawnictwo Politechniki Częstochowskiej, Częstochowa 2013 (in Polish).

[17] Finemet Alloys, Technical Bulletin, Metglas, www.metglas.com.

[18] Soft magnetic alloys and cores, Gammamet 421/501, GAMMA Research \& Production Enterprise, Ekaterinburg.

[19] IEC Standard: Magnetic materials, part 3: Methods of measurement of specific total losses of magnetic sheet and strip by means of a single sheet tester, International Electrotechnical Commission, Publication 404-3-1997, 1997.

[20] K. Sokalski, J. Szczygłowski, M. Najgebauer, W. Wilczyński, COMPEL 26, 640 (2007).

[21] M. Najgebauer, Prz. Elektrotechniczny 12, 213 (2008).

[22] K. Sokalski, J. Szczygłowski, Acta Phys. Pol. A 115, 920 (2009).

[23] K. Sokalski, J. Szczygłowski, Acta Phys. Pol. A 117, 497 (2010).

[24] M. Najgebauer, in: Proc. XII Int. Ph.D. Workshop OWD'2010, Wista (Poland), Vol. 28, Ed. G. Kłapyta, Conference Archives PTETiS, Gliwice 2010, p. 477.

[25] J. Szczygłowski, P. Kopciuszewski, K. Chwastek, M. Najgebauer, W. Wilczyński, Prz. Elektrotechniczny 4, 45 (2010) (in Polish).

[26] W.J. Yuan, F.J. Liu, S.J. Pang, Y.J. Song, T. Zhang, Intermetallics 17, 278 (2009). 